\title{
Chewing gum for declining ileus and accelerating gastrointestinal recovery after appendectomy
}

\author{
Manal Hamed Mahmoud ${ }^{a, *}$, Safaa Hussein Mohammad \\ aMedical-Surgical Nursing, Faculty of Nursing, Benha University, Tahch, Elqualubia Governorate 13741, Egypt \\ ${ }^{b}$ Medical-Surgical Nursing, Faculty of Nursing, Zagazig University, Facous, El Sharkia Governorate 44684, Egypt
}

Received: 19 February 2018; Accepted: 28 March 2018; Published: 20 December 2018

\begin{abstract}
Objective: lleus usually occurs after abdominal surgery and is allied with complication and delays recovery. It is hypothesized that chewing gum reduces postoperative ileus by improving early recovery of gastrointestinal function. This study aimed to explore whether chewing gum after appendectomy accelerates the recovery of gastrointestinal function.

Methods: Randomized control trial was used in this study. This study was conducted in the General Surgery Department at Zagazig University Hospital. A total of 240 patients undergoing appendectomy were involved in this study; they were divided into the chewing sugar-free gum group (120) and the control group (120). Two tools were utilized in this study. Tool I: Structured Interviewing Schedule: part 1: assessment of personnel characteristics. Part 2: assessment of anthropometric measurements of the studied subjects as well as pre- and intraoperative indicators of them. Tool II: postoperative assessment sheet: assessed postoperative parameters of the intestinal function, occurrence of postoperative ileus, and related symptoms were assessed among studied participants.

Results: There were highly significant statistical differences in the time of resumption of gastrointestinal functions and postoperative ileus symptoms between the two groups $(P<0.001)$, which was significantly shorter in the chewing gum group compared to the control group.

Conclusions: The use of chewing gum is a useful and cheap method that can be employed to cut down the time to recover and accelerate normalization of gastrointestinal function. Chewing sugar-free gum after abdominal surgery is recommended to be added to the protocol of nursing care in the surgery units as well as its involvement in the nursing curriculum.
\end{abstract}

Keywords: appendectomy • chewing gum • gastrointestinal recovery • ileus

(c) Shanxi Medical Periodical Press.

\section{Introduction}

After the majority of surgeries, the activity of the gastrointestinal tract is inhibited and this causes abdominal distention, a buildup of gas, nausea, and vomiting. Extended and large abdominal surgery leads to more harshness of this disruption. ${ }^{1}$ Paralytic ileus is a critical disruption arising following abdominal surgery and is defined as the delayed regain of the synchronized intestinal motility. This disturbance makes the progress delayed and increases the hospital days of stay. ${ }^{2}$ Apart from the fiscal concerns, the patient's lack of comfort and

* Corresponding author.

E-mail:dr.manalh@yahoo.com (M.H. Mahmoud). other problems such as the hospital-acquired infections are major concerns. ${ }^{3}$

Postoperative ileus occurs due to the drop of intestinal movement and the reduction of the activity of the parasympathetic nervous system. ${ }^{4}$ Ileus occurs in cases of opioid and drug interaction and abdominal operations, especially in surgeries with extreme manipulation, and transiently contribute to impeding peristalsis (bowel movement); the related mechanism is possibly dysfunction in the parasympathetic system (inhibitory neurons) activity. $^{5}$

Postoperative ileus increases to be a cause of morbidity and the primary reason for the extended hospital

O Open Access. (C 2018 Manal Hamed Mahmoud, Safaa Hussein Mohammad, published by Sciendo. (c) BY-NC-ND This work is licensed under the Creative Commons Attribution NonCommercial-NoDerivatives 4.0 License. 
days of stay post abdominal surgery such as an appendectomy. The useful and safe encouragement of the improvement of gastrointestinal function post abdominal operation and avoidance of postoperative problems have initiated a prevalent apprehension between medical and nursing staff. ${ }^{6}$ Ileus resolution is habitually defined by the passage of flatus (gas) or feces or both. These are signs that intestinal function is being restored to normal, and the end points of postoperative ileus are usually measured. ${ }^{7}$

At this moment, a number of techniques have been deliberated for the lessening of the postoperative ileus phase, among which one can pass on to employ epidural anesthesia, the diminution of utilizing narcotic perfusion by recommending non-steroidal anti-inflammatory drugs, laparoscopic surgery, and using high carbohydrate drinks post the operation. ${ }^{8}$ Numerous studies sustain the belief that chewing gum improves postoperative ileus in general. ${ }^{9}$ Chewing gum can increase the bowel motility as it directly activates the cephalic-vagal reflex, which in turn activates intestinal myoelectric motion, and indirectly stimulates the secretion of gastrointestinal hormones that increase the release of saliva and pancreatic juice. This reaction leads to likewise humeral and nervous activation of bowel motility. ${ }^{10}$

A number of theories have been proposed for chewing as a type of sham feeding that enhances the plasma concentration of gastrin, neurotensin, pancreatic polypeptide, and duodenal alkaline secretion. ${ }^{11}$ The mechanism of improved revival from postoperative gastrointestinal dysfunction with the assistance of chewing gum is thought to be the cephalic-vagal stimulation of digestion, which enhances the capability of neural and humoral factors that operate on different portions of the gastrointestinal tract. ${ }^{12}$ Chewing gum proceeds by stimulating intestinal motility coupled with bowel motility that causes early return of bowel sounds, a passage of flatus, and arrival of appetite. ${ }^{13}$

It is accentuated that adding together to postoperative nursing concern, nurses should also utilize consistent, cheap, accessible, and valuable practices in order to condense the negative property of problems that patients face. Practices such as postoperative standing up in the early hours, gum chewing, and early fluid drinking, which are accessible, inexpensive, and steadfast after abdominal surgeries are utilized traditionally in recent times. These approaches are considered to reduce the recovery period and length of time to discharge from the hospital. ${ }^{14}$ Consequently, gum chewing as an artificial feeding manner named as sham feeding is utilized in order to begin bowel movements in a short time in various studies; therefore, the researchers have taken up this study to evaluate the effect of chewing gum on gastrointestinal function after appendectomy surgery.

Following abdominal surgeries, the reduction of gastrointestinal system motility and the alteration of dietary habits may cause progress of ileus symptoms, such as nausea, vomiting, abdominal distension, and hiccup, which leads to severe pain for the patient/individual after operation. ${ }^{15}$ Postoperative ileus is the most major postabdominal surgery crisis that extends the interval of hospital stay, causes pain and distension, hinders oral feeding, causes respiratory troubles, and increases hospital expenses. ${ }^{16,17}$

Consequently, resumption of the intestinal function is an essential feature that necessitates appropriate awareness. ${ }^{18}$ Chewing gum can augment bowel movements and decrease the temporary phase of paralysis. ${ }^{19}$ Using chewing gum as one of the nonpharmacological interferences and an economical method that can be used to activate the stomach improves gastric secretion, enhances peristaltic bowel movements, and finally accelerates retrieval of intestinal function. ${ }^{20}$

Although working with the patient undergoing abdominal operation it is the duty of the nurse to avert the postoperative ileus, there are many nonpharmacological treatments, such as early enteral nutrition, early mobilization, and psychological preoperative training, and among them, the utilization of chewing gum has appeared as a latest, effortless, willingly obtainable, and inexpensive modality for lessening postoperative ileus. ${ }^{13}$ This intervention has been revealed to be successful in the postoperative time of gastrointestinal surgery. ${ }^{21,22}$ Moreover, the outcome of such a topic in our Egyptian context is necessary; thus, this study is carried out to explore whether chewing gum after appendectomy accelerates the recovery of gastrointestinal function. Appendectomy patients who chew sugar-free gum recover their gastrointestinal functions earlier than those who do not.

\section{Methods}

\subsection{Subjects and methods}

Randomized control trial was used to carry out this study in the General Surgery Department at Zagazig University Hospital.

\subsubsection{Sample}

A-Type: a purposive sample of postoperative appendectomy patients who met the criteria for inclusion in this study. 
B-Size: the sample size was calculated based on the previous year census report of the Surgical Department at Zagazig University Hospital. The total number of patients undergoing appendectomy was $600 .{ }^{23}$ The sample size was calculated by the following formula. ${ }^{24}$

$$
n=\frac{N}{1+N(e)^{2}}
$$

where, $n=$ sample size, $N=$ total population number (600), and $e=$ margin error (0.05).

A total of 240 patients undergoing appendectomy surgery were employed in this study. After the performance of appendectomy surgery, patients were randomly separated into the chewing gum group (120 patients) and the control group (120 patients). We made a simple randomization by assigning the first patient to the chewing gum group and the next one to the control group, this being recurrent over and over until 240 cases were gained.

\subsubsection{Tools for data collection}

To attain the aim of this study, the following two tools were utilized for data collection.

Tool I: Structured interviewing schedule; consisted of two parts: prepared by the investigators after appraisal of the current literature. . $13,25,26$

Part 1: it included the following five items to assess personal characteristics of the studied participant: age, sex educational level, occupation, and residence.

Part 2: it involved items to assess anthropometric measurements of studied participants such as weight, height, body mass index, as well as items to assess preand intraoperative indicators of them such as duration of complaining, fasting before surgery, and duration of surgery.

Tool II: Post-Operative Assessment Sheet: adapted from the previous literature. ${ }^{13,25,26}$

It integrated nine components for assessing the postoperative parameters of intestinal function, including time of first intestinal sound, time of first passage of flatus, time of first feeling of hunger, time of first defecation, and the time of hospital stay by hour, as well as occurrence of postoperative ileus and related symptoms among studied participants such as abdominal distension, nausea, and vomiting after appendectomy.

\subsubsection{Inclusion criteria}

The inclusion criteria were as follows: fasting for at least 8 hours before surgery, proper vital signs, no uncommon complications during surgery, and no medical disorders, such as hypothyroidism, diabetes, and neuromuscular disorders. Patients with postoperative complications and patients with intra-operative complications (severe adhesions, excessive manipulation of the intestine, blood transfusion, and injury to bowel or bladder) were included.

\subsubsection{Exclusion criteria}

The exclusion criteria were as follows: incorporated history of analgesic use, especially opioids, fluid, and electrolyte imbalance, pancreatitis or peritonitis, history of abdominal surgery, no willingness to cooperate, intra- and postoperative complications, and inability to chew gum.

\subsubsection{Tool validity}

Tools were evaluated by three experts in medicalsurgical nursing, and two surgeons tested the content validity. The tools were adapted according to the experts' proposition.

\subsubsection{Reliability}

Reliability of tool I was tested through a pilot study by using Cronbach's $\alpha(\alpha=0.87)$. Tool II had an internal consistency reliability estimate of 0.84 , and hence the questionnaire was found to be highly reliable.

\subsubsection{Ethical considerations}

An ethical approval was obtained from the head of the General Surgery Department at Zagazig University Hospital, to obtain the official agreement to perform the study after amplifying the aim of the study.

Written informed consents were obtained from all patients included in the study after explaining the purpose of the study.

The study subjects reassured about the ambiguity, confidentiality, security, and secrecy of the gathered data, as well as they enlightened about their rights to reject sharing or withdraw from the study at any time.

\subsection{Pilot study}

A pilot study was conducted in $10 \%$ of the study subjects in order to test the appropriateness and significance of the study tools and to test simplicity of the planned questionnaire as well as to guesstimate the time needed to answer them. Then, the required adjustments were done, and these subjects prohibited from adding into those of study sample. 


\subsection{Field work}

(1) The fieldwork of this research was carried out from April 2017 to July 2017.

(2) A written informed consent was obtained from the participants who fulfilled the inclusion criteria, and they were given a justification on the aim of the study before participation.

(3) The study group and the control group were allocated by using a simple random method, and then, researchers collected their personal characteristics.

(4) Protocol of chewing gum was explained to the intervention group.

(5) Gum chewing was started 2 hours after surgery and continued every 2 hours for 15 minutes in duration excluding throughout sleeping until passing flatus.

(6) Each participant in both groups was assessed abdominally using a stethoscope to identify the intestinal sound every 1 hour and was asked to report immediately the time of either feeling an intestinal movement, passing flatus or stool, first time of feeling hunger, first time of passing flatus, and defecation time and document the time of hospital discharge.

(7) The collected data were coded, analyzed, and then the results were compared between the two groups.

\subsection{Statistical analysis}

Statistical Package for Social Sciences version 15 was used for data analysis. Data were presented using descriptive statistics as mean and SD for quantitative variables, frequencies, and percentages for qualitative variables. Qualitative variables were compared using the chi-square test, whereas $t$-test was used to compare mean scores between study and control groups. Statistical significance was considered at a $P$-value of $\leq 0.05$, and a $P$-value of $\leq 0.001$ was considered to be highly significant.

\section{Results}

Table 1 presents personnel characteristics of the studied participants in both intervention and control groups. It is seen that there was no significant statistical difference between intervention and control groups regarding their age, sex, educational qualification, residence, and occupational status.

Table 2 shows the studied participants' anthropometric measurements in both intervention and control groups, which reveals that there was no significant

\begin{tabular}{|c|c|c|c|c|c|c|}
\hline \multirow[t]{2}{*}{$\begin{array}{c}\text { Personal } \\
\text { characteristics }\end{array}$} & \multicolumn{2}{|c|}{$\begin{array}{l}\text { Intervention group } \\
\qquad(n=120)\end{array}$} & \multicolumn{2}{|c|}{$\begin{array}{l}\text { Control group } \\
\quad(n=120)\end{array}$} & \multirow[t]{2}{*}{$\chi^{2}$} & \multirow[t]{2}{*}{$P$} \\
\hline & No & $\%$ & No & $\%$ & & \\
\hline \multicolumn{7}{|l|}{ Age in years } \\
\hline 20 to $<25$ & 37 & 30.8 & 38 & 31.7 & \multirow{3}{*}{0.73} & \multirow{3}{*}{$>0.05$} \\
\hline 25 to $<30$ & 59 & 49.2 & 63 & 52.5 & & \\
\hline $30-36$ & 24 & 20.0 & 19 & 15.8 & & \\
\hline \multicolumn{7}{|l|}{ Sex } \\
\hline Male & 76 & 63.3 & 79 & 65.8 & \multirow{2}{*}{0.16} & \multirow{2}{*}{$>0.05$} \\
\hline Female & 44 & 36.7 & 41 & 34.2 & & \\
\hline \multicolumn{7}{|l|}{ Educational level } \\
\hline $\begin{array}{l}\text { Read and } \\
\text { write }\end{array}$ & 18 & 15.0 & 13 & 10.8 & \multirow{3}{*}{2.30} & \multirow{3}{*}{$>0.05$} \\
\hline Secondary & 55 & 45.8 & 49 & 40.8 & & \\
\hline University & 47 & 39.2 & 58 & 48.3 & & \\
\hline \multicolumn{7}{|l|}{ Residence } \\
\hline Rural & 79 & 65.8 & 76 & 63.3 & \multirow{2}{*}{0.16} & \multirow{2}{*}{$>0.05$} \\
\hline Urban & 41 & 34.2 & 44 & 36.7 & & \\
\hline \multicolumn{7}{|l|}{ Occupation } \\
\hline Work & 18 & 15.0 & 13 & 10.8 & \multirow{2}{*}{0.75} & \multirow{2}{*}{$>0.05$} \\
\hline No work & 102 & 85.0 & 107 & 89.2 & & \\
\hline
\end{tabular}

Table 1. Personal characteristics of the studied participants.

\begin{tabular}{lrrrrrr}
\hline Variables & $\begin{array}{c}\text { Intervention } \\
\text { group }(n=120)\end{array}$ & $\begin{array}{c}\text { Control group } \\
(n=120)\end{array}$ & $\begin{array}{c}\text { Independent } \\
\text { t-test }\end{array}$ & $P$ \\
\cline { 2 - 4 } & Mean & SD & Mean & SD & & \\
\hline \hline Weight & 74.81 & 16.87 & 75.71 & 13.97 & 0.71 & $>0.05$ \\
Height & 162.58 & 8.87 & 162.87 & 7.95 & 0.45 & $>0.05$ \\
$\begin{array}{l}\text { Body mass } \\
\text { index }\end{array}$ & 28.41 & 6.18 & 28.57 & 5.62 & 0.26 & $>0.05$ \\
\hline
\end{tabular}

Table 2. Mean score of anthropometric measurements of the studied sample.

statistical difference between the two groups regarding their weight, height, and body mass index.

Table 3 shows the studied participants' pre- and intraoperative indicators in both intervention and control groups, which reveals that there is no significant statistical difference between the two groups regarding their duration of complaint, fasting hours before surgery, and duration of surgery.

Table 4 indicates the resumption of bowel function measures between intervention and control groups, which illustrates that there are highly statistically significant differences between both groups regarding the first time of bowel sound, the passage of flatus, defecation, feeling of hunger, and length of hospital stay.

Table 5 shows that there are highly significant statistical differences between intervention and control groups concerning the occurrence of postoperative ileus and its related symptoms such as feeling of abdominal distention, nausea, and vomiting. 


\begin{tabular}{lcccccc}
\hline Variables & \multicolumn{2}{c}{$\begin{array}{c}\text { Intervention } \\
\text { group }(n=120)\end{array}$} & $\begin{array}{c}\text { Control group } \\
(n=120)\end{array}$ & $\begin{array}{c}\text { Independent } \\
\text { t-test }\end{array}$ & $P$ \\
\cline { 2 - 4 } & Mean & SD & Mean & SD & & \\
\hline \hline $\begin{array}{l}\text { Duration of } \\
\text { complaint } \\
\text { (in hours) }\end{array}$ & 23.49 & 6.05 & 22.66 & 4.69 & 1.80 & $>0.05$ \\
$\begin{array}{l}\text { Fasting hours } \\
\text { before surgery }\end{array}$ & 42.33 & 5.80 & 40.92 & 5.61 & 1.92 & $>0.05$ \\
$\begin{array}{l}\text { Duration of } \\
\text { surgery } \\
\text { (in minutes) }\end{array}$ & 6.24 & 1.29 & 6.22 & 1.03 & 0.17 & $>0.05$ \\
\hline
\end{tabular}

Table 3. Pre- and intraoperative indicators of intervention and control groups.

\begin{tabular}{|c|c|c|c|c|c|c|}
\hline \multirow[t]{2}{*}{ Variables } & \multicolumn{2}{|c|}{$\begin{array}{l}\text { Intervention } \\
\text { group } \\
(n=120)\end{array}$} & \multicolumn{2}{|c|}{$\begin{array}{c}\text { Control } \\
\text { group } \\
(n=120)\end{array}$} & \multirow[t]{2}{*}{$\begin{array}{c}\text { Independent } \\
t \text {-test }\end{array}$} & \multirow[t]{2}{*}{$P$} \\
\hline & Mean & SD & Mean & SD & & \\
\hline $\begin{array}{l}\text { The first bowel } \\
\text { sounds (hours) }\end{array}$ & 10.05 & 1.44 & 15.22 & 2.02 & 22.81 & $<0.001^{*}$ \\
\hline $\begin{array}{l}\text { The first } \\
\text { passage of } \\
\text { flatus (hours) }\end{array}$ & 19.46 & 1.87 & 24.43 & 1.80 & 21.02 & $<0.001^{* *}$ \\
\hline $\begin{array}{l}\text { The first } \\
\text { defecation } \\
\text { (hours) }\end{array}$ & 19.74 & 1.84 & 31.03 & 8.28 & 14.54 & $<0.001^{* *}$ \\
\hline $\begin{array}{l}\text { The feeling of } \\
\text { hunger }\end{array}$ & 13.83 & 4.96 & 28.58 & 12.34 & 12.14 & $<0.001^{* *}$ \\
\hline $\begin{array}{l}\text { Hospital stay } \\
\text { (hours) }\end{array}$ & 28.81 & 12.13 & 35.63 & 7.32 & 5.27 & $<0.001^{\star *}$ \\
\hline
\end{tabular}

Table 4. Postoperative parameters in both groups. Note: * Statistically significant difference $(P<0.05)$;* Highly statistically significant difference $(P<0.001)$

\begin{tabular}{|c|c|c|c|c|c|c|c|c|c|c|}
\hline \multirow{3}{*}{ Variables } & \multicolumn{4}{|c|}{$\begin{array}{l}\text { Intervention group } \\
\quad(n=120)\end{array}$} & \multicolumn{4}{|c|}{$\begin{array}{c}\text { Control group } \\
(n=120)\end{array}$} & \multirow{3}{*}{$\chi^{2}$} & \multirow{3}{*}{$P$} \\
\hline & \multicolumn{2}{|c|}{ No } & \multicolumn{2}{|c|}{ Yes } & \multicolumn{2}{|r|}{ No } & \multicolumn{2}{|c|}{ Yes } & & \\
\hline & No & $\%$ & No & $\%$ & No & $\%$ & No & $\%$ & & \\
\hline $\begin{array}{l}\text { Abdominal } \\
\text { distension }\end{array}$ & 108 & 90.0 & 12 & 10.0 & 59 & 49.2 & 61 & 50.8 & $44.18<$ & $<0.001^{* *}$ \\
\hline Nausea & 111 & 92.5 & 9 & 7.5 & 69 & 57.5 & 51 & 42.5 & $68.37<$ & $<0.001^{* *}$ \\
\hline Vomiting & 113 & 94.2 & 7 & 5.8 & 58 & 48.3 & 62 & 51.7 & $54.87<$ & $<0.001^{* *}$ \\
\hline $\begin{array}{l}\text { Postoperative } \\
\text { ileus }\end{array}$ & 116 & 96.7 & 4 & 3.3 & 65 & 54.2 & 55 & 45.8 & $58.45<$ & $<0.001^{* \star}$ \\
\hline
\end{tabular}

Table 5. Distribution of postoperative ileus symptoms among studied participants.

Note: ** Highly statistically significant difference $(P<0.001)$

\section{Discussion}

Assessment of gastrointestinal function after abdominal surgeries is an important responsibility of the nurses. They should assess the function of the gastrointestinal tract by frequent auscultation of the bowel movement until the regain of ordinary peristaltic movement in all quadrants of the abdomen. The nurses have to be alert about signs related to decreased bowel movement, which include missing or deferred bowel sounds, abdominal distention, and stoppage to pass gas or stool. ${ }^{27}$

Results of this study demonstrate that there are no significant statistical differences between the chewing gum group and the control group regarding their personal characteristics, such as age, sex, educational level, residence, and occupation. Also, there is no significant difference between the intervention and control groups regarding their anthropometric measurements such as weight, mass index, as well as their pre- and intraoperative indicators such as duration of complaining, fasting hours before surgery, and time of surgery. These findings mean that the intervention group and the control group are homogenous and comparable. Our findings are congruent with the results of previous studies that found no significant discrepancy between the control and sugar-free gum chewing groups in terms of demographic characteristics, duration of surgery, body mass index, and fasting time before the surgery.

Pertaining to postoperative parameters, findings of this study indicated that there were significant statistical differences between the chewing gum and control groups regarding postoperative parameters of the gastrointestinal function, whereas the mean time of the onset of bowel sound, the onset of the gas passage, the onset of defecation, the first feeling of hunger, and the length of hospital stay was significantly lower in the chewing gum group than in the control group. These findings recommended that chewing gum enhances gastrointestinal function among patients with appendectomy. According to the significant literature, gum chewing promotes food digestion and secretion of salivary and hepatic glands through vagus nerve stimulation, and thus gum chewing directly augments intestinal stimulation through the release of gastrointestinal hormones that increase the secretion of saliva and pancreatic juices and subsequently promotes ileus recovery. ${ }^{11}$

The findings of this study are congruent with numerous relevant studies. The first was a metaanalysis of randomized clinical trials assessing the effect of gum chewing on gut function after elective colorectal surgery; ${ }^{29}$ the second was meta-analysis to examine if gum chewing reduces postoperative ileus; ${ }^{30}$ the third was a similar study on patients with open appendectomy to study if chewing gum reduces postoperative ileus after open appendectomy, and they found that the first passage of flatus occurred earlier 
in the chewing gum group than in the control group; the first bowel movement occurred earlier in the chewing gum group than in the control group; and hospital stay was shorter in the chewing gum group than in the control group ${ }^{31}$. Also, two meta-analyses that assessed the gum chewing as another simple possible method for more rapid enhancement of postoperative gastrointestinal function showed reduction in time of hearing first intestinal sound, passing flatus, defecation, and length of hospital stay. ${ }^{22,32}$ Two studies examined the effect of gum chewing on postoperative ileus and inflammation in colorectal surgery, ${ }^{33,34}$ as well, and one study determined the effect of gum chewing on postoperative ileus after cholecystectomy surgery, ${ }^{19}$ demonstrating that chewing the sugar-free gum will lead to the decrease of the time to gas passing, defecation, and bowel sound hearing time. Moreover, another study noted that the time to passage of first flatus was 7 hours earlier than those in the "typical care" control group; the time to passage of feces occurred on average 9 hours shorter in the intervention group; the average duration of hospital stay was shorter in the intervention group compared to the control group; and the first intestinal sounds were heard earlier in the intervention group than in the control group. ${ }^{35}$

In contrast to the results of this study, in a study carried out to evaluate the effect of chewing sugared gum in combination with early enteral feeding on the recovery of gastrointestinal (GI) function after major colorectal surgery, it was demonstrated that there was no significant difference in time to tolerating a lowresidue diet, time to flatus, time to bowel movement, length of postoperative hospital stay, and postoperative complications. ${ }^{36}$ The discrepancy may be due to two main factors. The first factor is the starting of early enteral feeding, which activates the gastrointestinal motility. The second factor is the use of sugared gum in their study, which differs in its effect from sugarless gum. In addition, chewing gum composition; sugar-free gum uses sugar substitutes (e.g., sorbitol and xylitol). These sugar surrogates can improve gut function by causing a nonstimulant laxative effect. This represents another factor, which may influence gut motility in the gum-chewing group of patients. ${ }^{37}$

The findings of this study are supported by the findings of previous researchers regarding the feeling of hunger after appendectomy, which was faster among the chewing gum group than the control group. These studies concluded that the feeling of hunger was earlier among the chewing gum group in the other one. ${ }^{13,28,38}$ Also, one study discovered that the first hungry feeling was statistically significantly faster in favor of the study group contrasted to the control group. ${ }^{39}$ On the other hand, there is another study that found no significant difference between the gumchewing group and the other group with respect to time to start the diet. The discrepancy between these results and results of this study may be attributed to the differences that existed between sample, type, and time of surgery. ${ }^{25}$

Concerning ileus and its signs and symptoms, results of this study discovered that there was a significant statistical reduction in the occurrence of postoperative ileus and related symptoms such as nausea, vomiting, and distention favoring the chewing gum group than the other one. In this respect, a previous study pointed out that chewing gum improves recovery after open appendectomy by reducing postoperative ileus among appendectomy patients. ${ }^{31}$ These results are in line with many studies that conducted metaanalysis for the relation between chewing gum and postoperative ileus and demonstrated a significant effect suggesting that chewing gum following abdominal surgery offers benefits in reducing the time of postoperative ileus. ${ }^{22,30,40-42}$ In addition, one study noted that gum chewing reduced postoperative ileus and inflammation among colorectal surgery patients ${ }^{33}$ and another one reported a reduction of paralytic ileus following cholecystectomy by chewing gum. ${ }^{19}$

Some studies contradict the findings of this study. One study reported that there was no benefit to sugared chewing gum in comparison with no gum in patients undergoing major colorectal surgery managed with early feeding regarding postoperative pain, nausea, or appetite. ${ }^{36}$ Also, there are two previous studies that found no effect of sugared chewing gum for patient with colorectal surgery compared to no gum chewing in their study conducted for the effect of sugared chewing gum on the return of gastrointestinal function after major surgery. ${ }^{25,43}$ The negative results are possibly due to their practice of early enteral feeding, which may already fasten the postoperative recovery of gastrointestinal function. In addition, the pathophysiology of postoperative ileus may be different for various operative techniques and anatomical portions of the GI tract. ${ }^{44}$

\section{Conclusions}

Supported by the overall findings of this study, we conclude that chewing gum is an accessible, effortless, safe, harmless, cheap, and effective method in declining ileus and accelerating gastrointestinal recovery after appendectomy. 


\section{Recommendations}

Based on the findings of this study, the following recommendations are suggested:

(1) Chewing sugar-free gum should be added in the protocol of nursing care after appendectomy surgery in the surgery units.

(2) Involvement of chewing gum after appendectomy surgery into nursing curriculum.

(3) Conducting a further study for evaluating the effect of chewing gum on postoperative ileus

\section{References}

1. Rafati S, Mashayekhi F, Rafat, F, Ezati Rad R, Pilevarzadeh M. Factors associated with cesarean section selection among pregnant women running head: Cesarean section. J Curr Res Sci. 2014;2:938-942.

2. Maldini B, Novotny Z, Letica-Brnadić R, Brkljacić $A$, Bartolek D. Difficult airway management with bonfils fiberscope in case of emergency: acute abdomen with ileus. Acta Clin Croat. 2012;51:483-487.

3. Kinoshita Y, Saitoh T, Ishihara S. Paralytic ileus. Nihon Rinsho. 2012;70 Suppl 6:557-60.557-560 (in Japanese).

4. Marwah S, Singla S, Tinna P. Role of gum chewing on the duration of postoperative ileus following ileostomy closure has done for typhoid ileal perforation: A prospective randomized trial. Saudi J Gastroenterol. 2012;18:111-117.

5. Johnson MD, Walsh RM. Current therapies to shorten postoperative ileus. Cleve Clin J Med. 2009;76:641-648.

6. Jakkaew B, Charoenkwan K. Effects of gum chewing on recovery of bowel function following cesarean section: A randomized controlled trial. Arch Gynecol Obstet. 2013;288:255-260.

7. Vather R, Trivedi S, Bissett I. Defining postoperative lleus: results of a systematic review and global survey. J Gastrointest Surg. 2013;17:962-972.

8. Gustafsson UO, Hausel J, Thorell A, et al. Adherence to the enhanced recovery after surgery protocol and outcomes after colorectal cancer surgery. Arch Surg. 2011;146:571-517.

9. Vásquez W, Hernández AV, Garcia-Sabrido JL. Is gum chewing useful for ileus after elective colorectal surgery? A systematic review and metaanalysis of randomized clinical trials. J Gastrointest Surg. 2009;13:649-656. among abdominal surgery patients using a larger sample and different geographical areas in Egypt.

(4) Carry out health education programs to abdominal surgery patients about the effect of chewing gum post surgery.

\section{Conflicts of interest}

All contributing authors declare no conflicts of interest.
10. Fanning J, Valea FA. Perioperative bowel management for gynecologic surgery. Am J Obstet Gynecol. 2011;205:309-314.

11. Choi H, Kang SH, Yoon DK, et al. Chewing gum has a stimulatory effect on bowel motility in patients after open or robotic radical cystectomy for bladder cancer: A prospective randomized comparative study. Urology. 2011;77:884-890.

12. Ge W, Chen G, Ding YT. Effect of chewing gum on the postoperative recovery of gastrointestinal function. Int J Clin Exp Med. 2015; 8:11936-11942.

13. Nimarta, Vir Singh, N. Shruti, N. and Gupta, R.(2013). Effectiveness of chewing gum on bowel motility among the patients who have undergone Abdominal Surgery.

14. International Council of Nurses. Closing the gap: From evidence to action-the international nurses day kit 2012. http://www.icn.ch/publications/2012closing-the-gap-from evidence to action. Accessed January 25, 2012.

15. Akyolcu, N. 2015. Postoperative nursing care. In: Aksoy, G., Kanan, N., Akyolcu, N. editors. Surgical Nursing I. Istanbul: Nobel Medical Bookstores; 2012. 357-358.

16. Hayes D. Stoma care nursing implications of postoperative ileus: a literature review. J Stomal Ther. 2012;32:18-20.

17. Mohsenzadeh Ledari F, Barat S, Delavar MA, Banihosini SZ, Khafri S. Chewing sugar-free gum reduces ileus after cesarean section in nulliparous women: a randomized clinical trial. Iran Red Cres Med J. 2013;15:330-334.

18. Ge W, Chen G, Ding YT. Effect of chewing gum on the postoperative recovery of gastrointestinal function. Int J Clin Exp Med. 2015;8: 11936-11942. 
19. Pilevarzadeh M. Effect of gum chewing in the reduction of paralytic ileus following cholecystectomy. Biomed Pharmacol J. 2016;9:405-409.

20. Hitti M. Chewing gum may shorten hospital stays after colon surgery. http://www.webmd.com/colorectal-cancer/news/20060221/gum-may-aid-colonsurgery-recovery. Accessed January 25, 2012.

21. Wang XJ, Chi P. Effect of chewing gum on the promotion of intestinal function recovery after colorectal surgery: a meta-analysis. Zhonghua Wei Chang Wai Ke Za Zhi. 2013;16:1078-1083 (in Chinese).

22. Yin Z, Sun J, Liu T, Zhu Y, Peng S, Wang J. Gum chewing: another simple potential method for more rapid improvement of postoperative gastrointestinal function. Digestion. 2013;87:67-74.

23. Zagazig University Hospital statistical Census Center. Surgical department census report. 2016.

24. Yamane T. Statistics an Introductory Analysis. 2nd ed. New York: Harper and Row.1967

25. Lim P, Morris OJ, Nolan G, MooreS, Draganic B, Smith S. Sham feeding with chewing gum after elective colorectal resectional surgery: a randomized clinical trial. Ann Surg. 2013;257:1016-1024.

26. Rashad WA, AL Yousef SA. Effect of sugarless gum chewing on intestinal movement after cesarean section. Life Sci J. 2013;10:3257-3261.

27. Murray SS, Mckinne ES. Foundation of Maternal and Newborn Health Nursing. 6th ed. USA: Elsevier Health Sci. 2014:409.

28. Ibrahim HA, Mohamady $\mathrm{SH}$. The 6th international scientific conference of faculty of nursing. Benha University. 2013.

29. Parnaby CN, MacDonald AJ, Jenkins JT. Sham feed or sham? A meta-analysis of randomized clinical trials assessing the effect of gum chewing on gut function after elective colorectal surgery. Int $\mathrm{J}$ Colorectal Dis. 2009;24:585-592.

30. Noble EJ, Harris R, Hosie KB, Thomas S, Lewis SJ. Gum chewing reduces postoperative ileus? A systematic review and meta-analysis. Int J Surg. 2009;7:100-105.

31. Ngowe MN, Eyenga VC, Kengne BH, Bahebeck J, Sosso AM. Chewing gum reduces postoperative ileus after open appendectomy. Acta Chir Belg. 2010;110:195-199.

32. Li S, Liu Y, Peng Q, Xie L, Wang J, Qin X. Chewing gum reduces postoperative ileus following abdominal surgery: a meta-analysis of 17 randomized controlled trials. J Gastroenterol Hepatol. 2013;28:1122-1132.
33. van den Heijkant TC, Costes LM, van der Lee DG, et al. Randomized clinical trial of the effect of gum chewing on postoperative ileus and inflammation in colorectal surgery. Br J Surg. 2015;102:202-211.

34. Short V, Herbert G, Perry R, et al. Chewing gum for postoperative recovery of gastrointestinal function. Cochrane Database Syst Rev. 2015;2:CD006506.

35. Pereira Gomes Morais E, Riera R, Porfírio GJ, et al. Chewing gum for enhancing early recovery of bowel function after caesarean section. Cochrane Database Syst Rev. 2016;10:CD011562.

36. Zaghiyan K, Felder S, Ovsepyan G, et al. A prospective randomized controlled trial of sugared chewing gum on gastrointestinal recovery after major colorectal surgery in patients managed with early enteral feeding. Dis Colon Rectum. 2013;56:328-335.

37. Tandeter $\mathrm{H}$. Hypothesis: Hexitols in chewing gum may play a role in reducing postoperative ileus. Med Hypotheses. 2009;72:39-40.

38. Ledari FM, Barat S, Delavar MA. Chewing gums has stimulatory effects on bowel function in patients undergoing cesarean section: a randomized controlled trial. Bosn J Basic Med Sci. 2012; 2:265-268.

39. Mansour SE, Elnegeri MA, Ibrahim AA. Chewing gum after cesarean section: it's effect on regaining intestinal function. IOSR J Nurs Health Sci. 2016;5:75-83.

40. Purkayastha S, Tilney HS, Darzi AW, Tekkis PP. Meta-analysis of randomized studies evaluating chewing gum to enhance postoperative recovery following colectomy. Arch Surg. 2008;143:788-793.

41. Fitzgerald JE, Ahmed I. Systematic review and metaanalysis of chewing-gum therapy in the reduction of postoperative paralytic ileus following gastrointestinal surgery. World J Surg. 2009;33:2557-2566.

42. Li S, Liu Y, Peng Q, Xie L, Wang J, Qin X. Chewing gum reduces postoperative ileus following abdominal surgery: a meta-analysis of 17 randomized controlled trials. J Gastroenterol Hepatol. 2013;28:1122-1132.

43. Forrester DA, Doyle-Munoz J, McTigue T, D’Andrea $S$, Natale-Ryan A. The efficacy of gum chewing in reducing postoperative ileus: a multisite randomized controlled trial. J Wound Ostomy Continence Nurs. 2014;41:227-232.

44. Luckey A, Livingston E, Taché Y. Mechanisms and treatment of postoperative ileus. Arch Surg. 2003;138:206-214.

How to cite this article: Mahmoud $\mathrm{MH}$, Mohammad $\mathrm{SH}$. Chewing gum for declining ileus and accelerating gastrointestinal recovery after appendectomy. Front Nurs. 2018; 4: 277-284. https://doi.org/10.1515/fon-2018-0038. 